\title{
Investigation of extended depth-of-field f/8 camera with optimized cubic phase mask and digital restoration
}

\author{
Po-Sheng Chiu ${ }^{1,2 *}$, Kurt Vonmetz ${ }^{2}$, Federico Canini ${ }^{2}$, and H. Paul Urbach $^{1}$ \\ ${ }^{1}$ Optics Research Group, Dept. of Imaging Physics, Delft University of Technology, Lorentzweg 1, 2628 CJ, Delft, the Netherlands \\ ${ }^{2}$ Datalogic IP Tech S.r.1., Via San Vitalino 13, 40012, Calderara di Reno (BO), Italy
}

\begin{abstract}
An investigation of extended depth-of-field camera with optimized phase mask and digital restoration is presented. The goal of this paper is to implement the wavefront coding technique without affecting much of the original design, and the design has taken the complexity of imaging system into consideration. The optimized strength of cubic phase mask (CPM) is based on the analytical optimal solution for the task-based imaging system [J. Opt. Soc. Am. A 25, 1064 (2008)]. The noisy intermediate images of CPM system with highest spatial frequency of interest can be effectively restored by vector-based RichardsonLucy algorithm. Restoration from the system with generalized CPM produces precise image position than the system with CPM does. In general, the CPM system procures modulation transfer function higher than 0.195 in the whole depth-of-field, and the mean squared error of the restored images are less than $5 \%$.
\end{abstract}

\section{Introduction}

Wavefront coding involves modification of the wavefront such that the imaging system is less sensitive to defocus, the cubic phase mask (CPM) is initially chosen [1]. The intermediate images of the wavefront coded system are blurred and it can be restored with a single kernel [2]. Moreover, the analytical expressions for the modulation transfer function (MTF) of system with CPM are given in the literature [3]. CPMs for extending depth-of-field (DOF) has been used in applications, such as, iris recognition [4], and microscopy [5]. In this paper, we implement CPM in a $\mathrm{f} / 8$ camera without affecting much of the original design. The challenge of the application is to capture a sufficiently good image in the range of DOF.

\section{Image defocus, formation, restoration and evaluation}

We choose to place CPM in the exit pupil plane has not only the design purpose that the total amount of light capture in the camera will pass through the exit pupil, but also the practical reason that the exit pupil is right in front of the compound lens of the system. The object distance is defined as the the distance from object plane to first principal plane (FPP), and the image distance is defined as the distance between second principal plane (SPP) and image plane. The focal plane of the camera is designed at $170 \mathrm{~mm}$ in front of FPP, hence by the thick-lens model we can calculate the location of image plane. The path length error $W$ in the normalized exit pupil plane $(x, y)$ can be determined by subtracting the ideal phase distribution from the out of focus phase distribution. The location of the image plane in the camera is fixed, and the object distance is varied. Therefore, $W$ is given by

$$
W(x, y)=\frac{1}{2}\left(\frac{1}{\left(\frac{1}{f}-\frac{1}{\bar{d}_{o}}\right)^{-1}-d_{\text {exit }}}-\frac{1}{\left(\frac{1}{f}-\frac{1}{d_{o}}\right)^{-1}-d_{\text {exit }}}\right)\left(x^{2}+y^{2}\right),
$$

where $f$ is the effective focal length, $\widetilde{d_{o}}(170 \mathrm{~mm})$ is the focal plane of the object, $d_{o}$ is the arbitrary object plane between $50 \mathrm{~mm}(1.203 \lambda)$ and $300 \mathrm{~mm}(-0.231 \lambda)$, and $d_{\text {exit }}$ is the distance from SPP to exit pupil. Image formation ( $I$ ) by a spatially invariant system under incoherent illumination can be written as the convolution of its PSF with the object $(O)$ by the following

$$
h * O=I \leftrightarrow H \cdot G_{o}=G_{i},
$$

where $h$ is the system's PSF, $*$ is the convolution operator, and noise usually appears in detected images; the capital letters correspond to the spatial frequency by the Fourier transformation, and the $H$ is the system optical transfer function (OTF), which equals to the Fourier transform of the PSF. The PSF of an incoherent system is given as $h(x, y, W)=\left|\iint p(x, y) e^{-i 2 \pi(x u+y v)} d x d y\right|^{2}$, where $u, v$ are the normalized spatial coordinates. The pupil function in our system $p(x, y)$ is written as

$$
p(x, y)=\exp \left\{i k\left[\phi(x, y)+W\left(x^{2}+y^{2}\right)\right]\right\} \operatorname{circ}(\mathrm{x}, \mathrm{y}),
$$

where $\phi$ is the modulation introduced by the phase mask, and $W$ is the defocus parameter as given in equ (1), and the circ function is defined as 1 inside the circular pupil and 0 outside of it. The modulation introduced by a CPM is expressed as $\phi_{C P M}(x, y)=\alpha\left(x^{3}+y^{3}\right)$, where $\alpha$ is the CPM parameter. Another commonly used mask is generalized cubic phase mask (GCPM), and given by $\phi_{G C P M}(x, y)=\alpha\left(x^{3}+y^{3}\right)+\beta\left(x^{2} y+x y^{2}\right)$, where $\beta=-3 \alpha$

* Corresponding author: p.chiu@tudelft.nl 
is usually assumed [6]. The favor of choosing GCPM is because it can produce a PSF which is approximately the same in each direction. The restored image will be the deconvolution of the intermediate image and the system PSF. Due to the ill-posed problem for inverse calculation, we investigate two methods for solving this issue in the restoration. The first one is the Wiener filter restoration,

$$
I_{W}=\mathcal{F}^{-1}\left\{\left(H_{d e c}{ }^{T} H_{d e c}+\lambda^{2} I\right)^{-1} H_{d e c}{ }^{T} G_{i}\right\},
$$

where $\mathcal{F}^{-1}$ stands for inverse Fourier transform, $H_{d e c}$ is the OTF restoration kernel (assume zero defocus OTF of the wavefront coded system), $T$ is the transpose of the matrix, and $\lambda$ is regularization parameter determined by L-curve [7]. The second method is the vector-based Richardson-Lucy algorithm [8], it calculates the direction of next point as the different between the current iteration and the previous iteration. If $x_{k}$ is the iterated point after $k$ iterations, $y_{k}$ the predicted point, $h_{k}$ the direction vector, and $\alpha_{k}$ the acceleration parameter then:

$$
y_{k}=x_{k}+\alpha_{k} h_{k}
$$

where $h_{k}=x_{k}-x_{k-1}, x_{k+1}=y_{k}+g_{k}, g_{k}=\psi\left(y_{k}\right)-y_{k} \cdot \psi$ is called the Richardson-Lucy function which expresses as $y_{k+1}=y_{k}\left(h \otimes \frac{I}{h * y_{k}}\right) \equiv \psi\left(y_{k}\right)$, where $\otimes$ is the correlation operator, and $h * y_{k}$ is referred to as the reblurred image after $k$ iterations. We use MTF as the quality assessment in optical design. Furthermore, we choose mean squared error (MSE) and structural similarity (SSIM) for the image evaluation metrics between the restoration image and the reference object.

\section{Simulation}

USAF 1951 test chart is our reference object, and we focus on group 1, element 4 which has the resolution of 2.83 line-pairs $/ \mathrm{mm}$. The following simulation is based on camera with pixel number $1024 \times 1024$, pixel size $3 \mu \mathrm{m}$ and $35 \mathrm{~dB}$ signal-to-noise ratio. Figure 1. displays restoration of the near field object with defocus of 1.203 $\lambda$ by using different phase masks and different algorithms. Vector-RL algorithm generate visually good images compare with the result from Wiener filter, besides, images form the conventional system cannot be restored with a single kernel. The effect of image shift of the CPM can be observed in row profile picture in Figure. 2. These artifacts result from the phase mismatch between convolution kernel and deconvolution kernel. From Table 1 , iterative method gives better result than Wiener filter does in near field, besides, system with GCPM provides higher SSIM and smaller MSE in same condition. Further, MTF of GCPM system is lower than CPM system because

\begin{tabular}{|c|c|c|c|c|}
\hline Phase mask & Defocus $(\lambda)$ & MTF & MSE & SSIM \\
\hline \multirow[t]{3}{*}{ CPM } & 1.203 & 0.425 & $0.040398 / 0.031871 / 0.035072$ & $0.3011 / 0.5110 / 0.44207$ \\
\hline & 0 & 0.284 & $0.009372 / 0.002035 / 0.002933$ & $0.4997 / 0.8270 / 0.7433$ \\
\hline & -0.2316 & 0.195 & $0.009635 / 0.002046 / 0.003124$ & $0.4854 / 0.8105 / 0.71439$ \\
\hline \multirow[t]{3}{*}{ GCPM } & 1.203 & 0.208 & $0.039017 / 0.018688 / 0.021098$ & $0.2103 / 0.5701 / 0.5441$ \\
\hline & 0 & 0.061 & $0.018683 / 0.012639 / 0.004558$ & $0.3369 / 0.6819 / 0.7251$ \\
\hline & -0.2316 & 0.035 & $0.018593 / 0.012655 / 0.004643$ & $0.3297 / 0.6955 / 0.7322$ \\
\hline
\end{tabular}
GCPM introduces more aberrations in the pupil function.

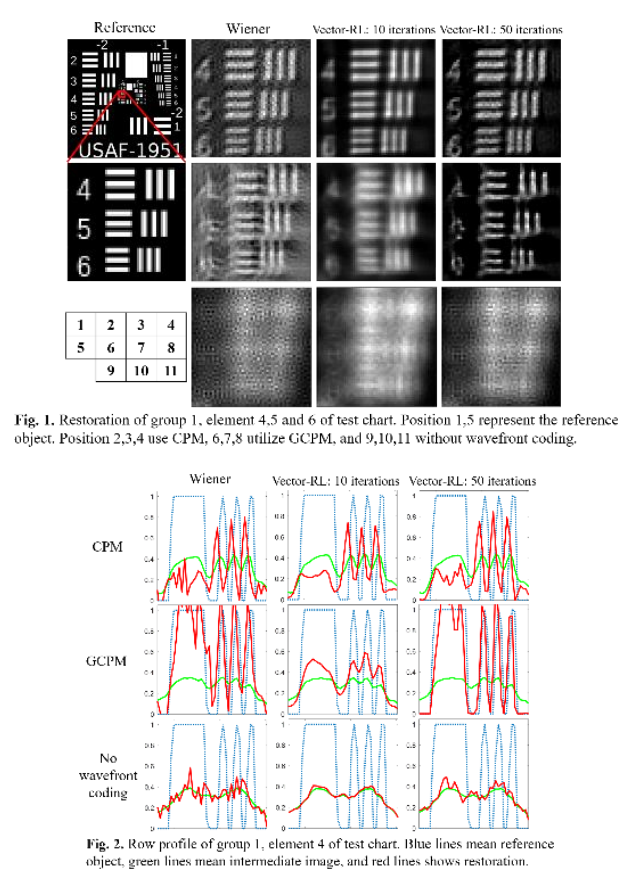

\section{Conclusion \& Outlook}

In conclusion, we investigate wavefront coding technique in a $\mathrm{f} / 8$ camera for extending DOF. The design has taken lens thickness, pupil planes, and principal planes into account. The image restoration shows that the noisy images with highest spatial frequency of interest can be effectively restored. Furthermore, due to the antisymmetric characteristic PSF of the CPM system, we further examine the possibility of using GCPM in the imaging system. The outcome provides us a solution to mitigate the image shift, however, system with CPM provide higher MTF values in the same range of DOF. The fabricated CPM and GCPM will be implemented in prove of concept experiment. Moreover, how to quantify the image artifacts and restore without image shift from the CPM system is the future direction.

This research has received funding from the European Union's Horizon 2020 research and innovation program under the Marie Skłodowska-Curie Grant Agreement No. 675745.

\section{References}

1. E. R. Dowski, Jr., W. T. Cathey, Appl. Opt. 34(11), 1859-1866 (1995)

2. W. T. Cathey and E. R. Dowski, Appl. Opt. 41(29), 6080-6092 (2002)

3. S. Bagheri et al., J. Opt. Soc. Am. A 25(5), 1051-1063 (2008)

4. S. Bagheri et al., J. Opt. Soc. Am. A 25(5), 1064-1074 (2008)

5. Y. Zhou et al., Opt. Express 26(6), 7563-7577 (2018)

6. S. Prasad et al., Proc. SPIE 5108, 1-12 (2003)

7. P. C. Hansen, Discrete inverse problem: insight and algorithms (SIAM, Philadelphia, 2010), chap.4

8. D. S. C. Biggs, M. Andrews, Appl. Opt. 36(8), 17661775 (1997) 